\title{
Emergency Department Blood Gas Utilization and Changes in Ventilator Settings
}

\author{
Haitham S Al Ashry MD, Jeremy B Richards MD, Daniel F Fisher MSc RRT, \\ Jeffrey Sankoff MD, Todd A Seigel MD, Lauren B Angotti MD, and Susan R Wilcox MD
}

\begin{abstract}
BACKGROUND: Mechanically ventilated patients increasingly spend hours in emergency department beds before ICU admission. This study evaluated the performance of blood gases in mechanically ventilated subjects in the emergency department and subsequent changes to mechanical ventilation settings. METHODS: This was a multi-center, prospective, observational study of subjects ventilated in the emergency department, conducted at 3 academic emergency departments from July 2011 to March 2013. We measured the rate of arterial blood gas (ABG) and venous blood gas (VBG) analysis, and we assessed the associations between the conditions of hypoxemia, hyperoxia, hypercapnia, or acidemia and changes to mechanical ventilator settings. RESULTS: Of 292 ventilated subjects, $17.1 \%$ did not have a blood gas sent in the emergency department. Ventilator changes were made significantly more frequently for subjects who had an ABG as the initial blood gas sent in the emergency department (odds ratio $2.70,95 \%$ CI $1.46-4.99, P=.002)$. However, findings of hypoxemia, hyperoxia, hypercapnia, or acidemia were not correlated with ventilator adjustments. CONCLUSIONS: In this prospective observational study of subjects mechanically ventilated in the emergency department, the majority had a blood gas checked while in the emergency department. While ABGs were associated with having changes made to ventilator settings in the emergency department, clinical findings of hypoxemia, hyperoxia, hypercapnia, and acidemia were not. Inattention to blood gas results may lead to missed opportunities in guiding ventilator changes in the emergency department. Key words: emergency department boarding; mechanical ventilation; overcrowding; emergency department to ICU transition; arterial and venous blood gas analysis. [Respir Care 2018;63(1):36-42. (C) 2018 Daedalus Enterprises]
\end{abstract}

\section{Introduction}

Nearly $60 \%$ of patients admitted to ICUs in the United States originate from emergency departments, and up to

\footnotetext{
Drs Al Ashry, Richards, Angotti, and Wilcox are affiliated with the Division of Pulmonary, Critical Care, and Sleep Medicine, Department of Medicine, Medical University of South Carolina. Dr Wilcox is also affiliated with the Division of Emergency Medicine, Medical University of South Carolina. Mr. Fisher is affiliated with Respiratory Care Services, Massachusetts General Hospital. Dr Sankoff is affiliated with the Department of Emergency Medicine, University of Colorado, School of Medicine, Denver Health Medical Center. Dr Seigel is affiliated with the Department of Emergency Medicine and Critical Care, Kaiser Permanente East Bay, Oakland and Richmond Medical Centers.
}

The authors have disclosed no conflicts of interest.

Correspondence: Haitham S Al Ashry MD, 96 Jonathan Lucas St, CSB Suite 816, Charleston, SC 29425. E-mail: alaashry@musc.edu

DOI: $10.4187 /$ respcare .05590
$40 \%$ are intubated and mechanically ventilated before ICU admission. ${ }^{1}$ While most ventilator changes occur during the first $48 \mathrm{~h}$ of ICU admission, ${ }^{2}$ a majority of mechanically ventilated patients in the emergency department receive no ventilator changes despite an average emergency department stay of 5-6 h before transfer to the ICU. ${ }^{3-5}$

A recent study of mechanically ventilated emergency department subjects found an association between an increased number of emergency department interventions after intubation and reduced mortality, ${ }^{6}$ demonstrating the need for ongoing monitoring and care of mechanically ventilated emergency department patients. In addition to hemodynamic disturbances, mechanically ventilated emergency department patients are at risk of hypoxemia, hyperoxia, hypercapnia, and acidemia. ${ }^{7}$ Several studies have shown hyperoxia to be associated with increased morbidity and mortality in critically ill subjects. ${ }^{8-10}$ In addition, close monitoring for hypercapnia and acidosis is important 
in mechanically ventilated patients, especially those receiving lung-protective ventilation. ${ }^{11}$

Although venous blood gases (VBGs) are preferred for many conditions encountered in the emergency department, ${ }^{12,13}$ arterial blood gases (ABGs) are indicated in recently intubated and ventilated patients. ${ }^{14}$ While pulse oximetry is valuable for monitoring adequate oxygenation, only data from $\mathrm{ABG}$ can be used to determine hyperoxia or define the $\mathrm{P}_{\mathrm{aO}_{2}}$ to $\mathrm{F}_{\mathrm{IO}_{2}}$ ratio. ${ }^{14-16}$ The use of blood gas analysis in mechanically ventilated emergency department patients has not been well described in the literature. In this study we explore patterns of blood gas analysis in the emergency department and evaluate associations between blood gas results and emergency department clinicians' management of ventilator settings.

\section{Methods}

This study was supported by institutional funds. At Rhode Island Hospital, the work was funded in part by an institutional development grant from the University Emergency Medicine Foundation. The data were collected at Denver General Hospital, Massachusetts General Hospital, and Rhode Island Hospital from 2011 to 2013. The data analysis and manuscript preparation were performed at the Medical University of South Carolina in 2016.

We performed a multi-center, prospective, observational, cohort study with data collected from emergency departments of 3 tertiary care academic centers, each with more than 100,000 emergency department visits annually, staffed with board-certified emergency physicians, emergency medicine residents, advanced practice providers, nurses, and respiratory therapists. All adult patients receiving invasive mechanical ventilation were eligible for enrollment. Exclusion criteria included death upon arrival or during emergency department course, or immediate transfer to the operating room from the emergency department. Patients without complete documentation regarding the duration of time ventilated in the emergency department and emergency department length of stay (LOS) were also excluded. All subjects were managed by the emergency medicine attending doctors for the duration of their time in the emergency department. The study protocol was approved by the institutional review boards where the study was conducted as well as in the institution where data were analyzed. Institutional review boards for all participating institutions approved the study protocols with waivers for informed consent.

Subjects were screened and enrolled upon emergency department presentation. To focus on blood gases obtained at the time of or subsequent to intubation, we restricted our cohort to subjects who were intubated prior to or upon arrival to the emergency department, defined as within 10 min of emergency department arrival. Subjects with miss-

\section{QUICK LOOK}

\section{Current knowledge}

Blood gas analysis is widely used in the ICU to make appropriate changes in ventilators' settings. In the recent years emergency departments' boarding times of mechanically ventilated patients have been increasing. The use of blood gas analysis to guide changes in ventilators' settings in this population has not been previously studied.

\section{What this paper contributes to our knowledge}

In the emergency departments of 3 tertiary care academic centers, the majority of mechanically ventilated subjects had a blood gas checked while in the emergency department. However clinical findings of hypoxemia, hyperoxia, hypercapnia, and acidemia were not associated with changes in ventilator settings.

ing data were excluded on a case-by-case basis. With regard to descriptive statistics for each variable, missing values were excluded from analyses, while for regression analysis if any variable was missing data, the entire subject was excluded from analysis.

Time ventilated in the emergency department was defined as the time of emergency department presentation for subjects initiated on ventilation prior to arrival, or time of intubation for the remainder of subjects, until the time of ICU admission. Emergency department LOS was defined as time from emergency department presentation to time of ICU admission. Subjects were classified by indication for intubation, including altered mental status without respiratory pathology, trauma, cardiac arrest, respiratory failure, neurologic events, airway edema, and other causes. Altered mental status was defined as encephalopathy or obtundation without primary central nervous system pathology, while neurologic events included cerebral vascular accidence, hemorrhage stroke, or seizures. Changes in ventilator settings included any change in the mode of ventilation, breathing frequency, tidal volumes, $\mathrm{F}_{\mathrm{IO}_{2}}$, driving pressure, or PEEP. Hypoxemia was defined as a $\mathrm{P}_{\mathrm{aO}}$ of $\leq 60 \mathrm{~mm} \mathrm{Hg}$ on $\mathrm{ABG}$, and hyperoxia was defined as a $\mathrm{P}_{\mathrm{aO}_{2}} \geq 300 \mathrm{~mm} \mathrm{Hg}$. Acidemia was defined as a $\mathrm{pH}$ of $\leq 7.20$ on ABG or VBG, and hypercapnia as a $\mathrm{P}_{\mathrm{CO}_{2}}$ of $\geq 60 \mathrm{~mm} \mathrm{Hg}$ by arterial or venous values. Lungprotective ventilation was defined as a tidal volume of $\leq 8 \mathrm{~mL} / \mathrm{kg}$ of predicted body weight, with full details published previously. ${ }^{5}$ None of the enrolling centers had specified protocols for blood gas ordering or mechanical ventilation management at the time of the study.

The primary outcome was the percentage of mechanically ventilated subjects who received any blood gas, ar- 
terial or venous, while in the emergency department. Secondary outcomes included evaluation of blood gas ordering for subjects' subgroups, including indication for intubation, subjects with hypotension, and subjects on vasopressors, and ventilator management with respect to blood gas values. We evaluated associations between hypoxemia or hyperoxia and changes in PEEP or $\mathrm{F}_{\mathrm{IO}_{2}}$, and associations between hypercapnia or acidemia and changes to tidal volume or respiratory frequency.

\section{Statistical Analysis}

Data were input into Microsoft Excel (Microsoft Corp., Redmond, Washington) and transferred to SPSS (version 24.0, IBM Corp, Armonk, New York) for statistical analysis. Data were visually inspected, and missing data were excluded on a case-by-case basis, with missing data as the primary parameter for exclusion. Outlier data points that were $>3$ standard deviations from the median were visually reviewed and rejected from analyses as manual entry errors. Descriptive statistics were used to determine the primary outcome. Subjects whose initial blood gas was arterial were included in analyses for outcomes for oxygenation parameters, while subjects with either initial ABGs or VBGs were included in analyses for outcomes for acid/base or ventilation parameters.

Binary logistic regression and Mann Whitney U test were used as appropriate for secondary outcomes. Continuous variables were reported as medians and standard deviations, and categorical variables were reported as numbers and percentages. Unadjusted odds ratios (ORs) were calculated to quantify differences in frequency of ventilator changes for different clinical parameters, including hypoxemia, hyperoxia, hypercapnia, acidemia, hypotension, vasopressor use, and indication for intubation. An alpha of $<0.05$ was considered statistically significant for all analyses. All $P$ values presented are for a two-tailed test.

\section{Results}

A total of 535 subjects were enrolled; 10 subjects were excluded as the duration of mechanical ventilation and stay in the emergency department were not fully documented, and an additional 233 were excluded for not being intubated upon arrival to the emergency department, resulting in a final study cohort of 292 subjects, 39\% of which were female. The average age was 57 y (range 19-96 y). Baseline demographics are outlined in Table 1. The median age for subjects intubated due to trauma $44.9 \mathrm{y}$ $( \pm 19.7 \mathrm{y})$ versus $60.7 \mathrm{y}( \pm 18.1 \mathrm{y})$ for all other indications $(P<.001)$. Average emergency department LOS and average emergency department ventilation time for the entire cohort was $282 \mathrm{~min}(\mathrm{SD} \pm 226 \mathrm{~min})$ and $262 \mathrm{~min}$ ( $\mathrm{SD} \pm 205 \mathrm{~min}$ ), respectively. Altered mental status was
Table 1. Baseline Characteristics

\begin{tabular}{lc}
\hline \hline \multicolumn{1}{c}{ Variable } & Participants \\
\hline Age, y (mean \pm SD) & $57 \pm 19.4$ \\
Female, $n(\%)$ & $97(33.2)$ \\
Emergency department LOS, min (mean \pm SD) & $282 \pm 226$ \\
Emergency department ventilator time, min & $262 \pm 205$ \\
$\quad$ (mean \pm SD) & \\
Indication of intubation, $n(\%)$ & $98(33.6)$ \\
$\quad$ Altered mental status & $73(25)$ \\
Trauma & $38(13)$ \\
$\quad$ Respiratory failure & $41(14)$ \\
$\quad$ Neuro & $27(9.2)$ \\
Cardiac arrest & $7(2.4)$ \\
Airway edema & $8(2.7)$ \\
Other & \\
\hline Total participiants: $N=292$. & \\
LOS = length of stay & \\
\hline
\end{tabular}

the most common indication for intubation, followed by trauma, neurologic events, and respiratory failure. In this cohort, $64.4 \%$ were ventilated with lung-protective ventilation, but 3 subjects (1\%) did not have recorded heights, precluding assessment for lung-protective ventilation.

Despite an average time of $>4 \mathrm{~h}$ of mechanical ventilation in the emergency department, 50 subjects (17.1\%) did not have any blood gas sent in the emergency department. Among those with blood gases, $22(9.0 \%)$ had a VBG but never an ABG. Of the 242 who had blood gases, 66 had a repeat gas in the emergency department, and 16 had 2 follow-up gases (Fig. 1). Only 23 subjects (9.5\%) had both VBGs and ABGs, and 143 subjects (59.1\%) had only ABGs. When analyzed by indication for intubation, subjects intubated due to trauma had a significantly higher OR of having an ABG (OR 5.35, $P=.032$, CI 1.1325.28) (Table 2). Subjects intubated for respiratory failure were not more likely to have an ABG performed as compared to subjects intubated for other indications (OR 1.75, $P=.48$, CI $0.36-8.45$ ). Subjects who did not have any blood gas checked had significantly longer median emergency department LOS at $273 \mathrm{~min}$ compared to $230 \mathrm{~min}$ $(P=.02)$.

In this study cohort, 137 (46.9\%) had an episode of hypotension, with $34(11.6 \%)$ receiving vasopressors. Of hypotensive subjects, only $26(19 \%)$ had an ABG. In hypotensive subjects receiving vasopressors, $38.2 \%$ did not have an ABG while in the emergency department.

The minority $(21 \%)$ of subjects had a change made to their ventilator settings in the emergency department, with 77 changes in ventilator settings made for 61 subjects. Subjects who had no ventilator changes made in the emergency department had a significantly longer median LOS in emergency department at $256 \mathrm{~min}$ versus $173 \mathrm{~min}$ 


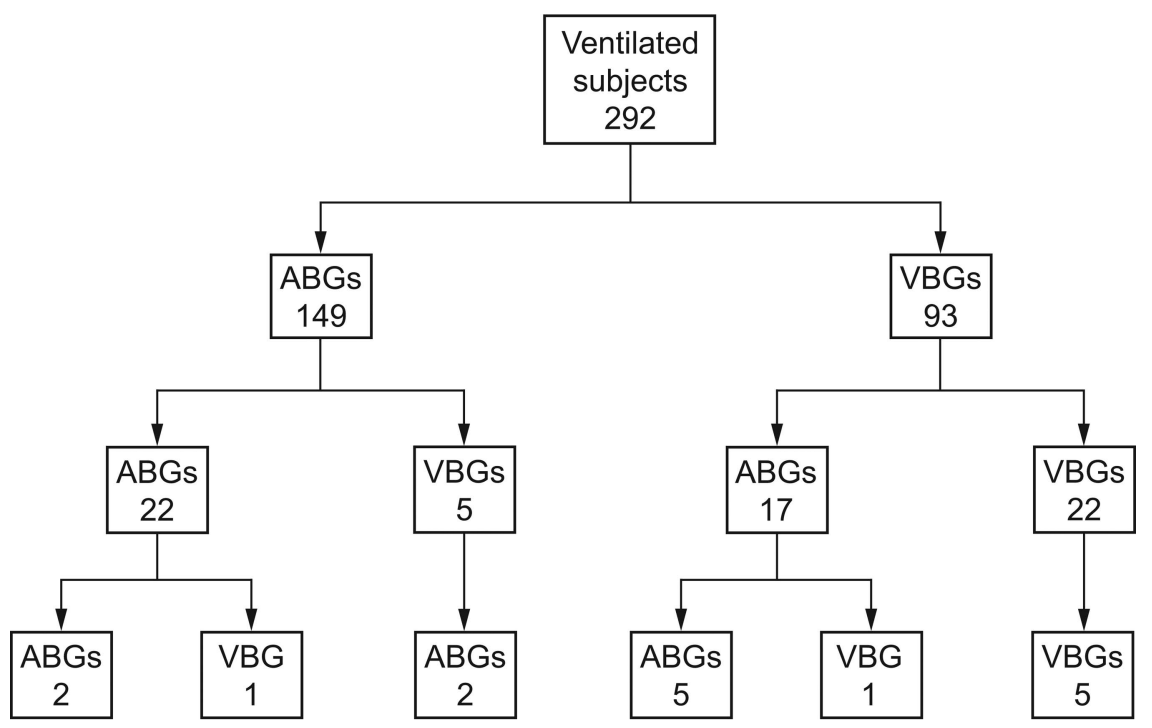

Fig. 1. Blood gases for mechanically ventilated patients in the emergency departments. First row shows initial gas, second row shows second gas, and third row shows third gas. ABG = arterial blood gas; VBG = venous blood gas.

Table 2. Odds Ratio of Ordering ABGs per Intubation Indication

\begin{tabular}{lcll}
\hline \hline Indication for Intubation & Odds Ratio & \multicolumn{1}{c}{$95 \% \mathrm{CI}$} & $P$ \\
\hline Altered mental status & 4.16 & $0.91-18.94$ & .068 \\
Trauma & 5.35 & $1.13-25.28$ & .032 \\
Respiratory failure & 1.75 & $0.36-8.45$ & .48 \\
Neuro & 1.14 & $0.22-5.81$ & .87 \\
Cardiac arrest & 4.04 & $0.75-21.72$ & .10 \\
Airway edema & 10 & $0.77-128.77$ & .07
\end{tabular}

$\overline{\mathrm{ABG}}=$ arterial blood gas

$(P=.001)$, and longer median emergency department ventilator time of $243 \mathrm{~min}$ versus $169 \mathrm{~min}(P=.002)$.

There was no correlation between having either an ABG or VBG and having a ventilator change made in the emergency department (OR 1.59, 95\% CI 0.69-3.71, $P=.34$ ). However, ventilator changes were made significantly more frequently for subjects who had an ABG as the initial blood gas (OR 2.70, 95\% CI 1.46-4.99, $P=.002$ ). Ventilator changes were less likely to be made when a VBG was the initial blood gas, as compared to sending an ABG (OR 0.36, 95\% CI 0.17-0.74, $P=.005$ ). Ventilator changes were as likely to be made when a VBG was the initial blood gas as compared to not sending a blood gas at all (OR 0.77, 95\% CI 0.28-2.14, $P=.79$ ).

Five subjects had hypoxemia on their initial ABG, with a $\mathrm{P}_{\mathrm{aO}_{2}}<60 \mathrm{~mm} \mathrm{Hg}$. All five subjects were ventilated with a PEEP of $5 \mathrm{~cm} \mathrm{H}_{2} \mathrm{O}$, but none had a change in PEEP. All were ventilated with a $\mathrm{F}_{\mathrm{IO}_{2}}$ of 1.0 , and 2 were on nonlung-protective ventilation (Table 3). Overall, 190 (65.1\%) subjects were ventilated with a $\mathrm{F}_{\mathrm{IO}_{2}}$ of 1.0 , and only 24 (12.6\%) had the $\mathrm{F}_{\mathrm{IO}_{2}}$ decreased. Among subjects who had
Table 3. Association Between Initial Blood Gas Results and Subsequent Ventilator Changes

\begin{tabular}{|c|c|c|c|}
\hline Blood Gas Parameter $(n)$ & $\begin{array}{l}\text { No. of Ventilator } \\
\text { Changes in } \\
\text { Emergency } \\
\text { Department } \\
\text { [(no. }(\%)]\end{array}$ & $\begin{array}{l}\text { Odds Ratio } \\
\text { (95\% CI) }\end{array}$ & $P$ \\
\hline \multicolumn{4}{|l|}{$\mathrm{P}_{\mathrm{aO}_{2}} \geq 60 \mathrm{~mm} \mathrm{Hg}(5)$} \\
\hline Change in PEEP & $0(0 \%)$ & NA & \\
\hline Change in $\mathrm{F}_{\mathrm{IO}_{2}}$ & $0(0 \%)$ & NA & \\
\hline \multicolumn{4}{|l|}{$\mathrm{P}_{\mathrm{aO}_{2}} \geq 300 \mathrm{~mm} \mathrm{Hg}(36)$} \\
\hline Change in PEEP & $0(0 \%)$ & NA & \\
\hline $\begin{array}{l}\text { Change in } \mathrm{F}_{\mathrm{IO}_{2}} \\
\mathrm{pH} \leq 7.2(70)\end{array}$ & $7(19.4 \%)$ & $1.37(0.52-3.60)$ & .61 \\
\hline Any change in tidal volume & $5(7.1 \%)$ & $4.10(0.95-17.60)$ & .055 \\
\hline Increase in tidal volume & $2(2.9 \%)$ & $1.64(0.27-10.02)$ & .63 \\
\hline Increase in breathing frequency & $9(12.9 \%)$ & $0.92(0.41-2.08)$ & .84 \\
\hline \multicolumn{4}{|l|}{$\mathrm{P}_{\mathrm{CO}_{2}} \geq 60$} \\
\hline Any change in tidal volume & $5(10.9 \%)$ & $7.1(1.6-30.8)$ & .01 \\
\hline Increase in tidal volume & $2(4.3 \%)$ & $2.84(0.46-17.50)$ & .25 \\
\hline Increase in breathing frequency & $3(6.5 \%)$ & $0.43(0.12-1.46)$ & .22 \\
\hline
\end{tabular}

$\mathrm{NA}=$ not applicable

ABGs as their initial blood gas, 36 (25.2\%) had hyperoxia, $35(97.3 \%)$ of whom were receiving a $\mathrm{F}_{\mathrm{IO}_{2}}$ of 1.0. A majority of these subjects $(80.6 \%)$ did not have the $\mathrm{F}_{\mathrm{IO}_{2}}$ decreased while in the emergency department.

Forty-six subjects $\left(15.8 \%\right.$ ) had a $\mathrm{P}_{\mathrm{CO}_{2}}$ of $>60 \mathrm{~mm} \mathrm{Hg}$, 14 by $\mathrm{ABG}$ and 32 by VBG. Of these 46 subjects, 6 had changes made to their ventilator settings in the emergency department, for a total of 8 discrete ventilator changes ( 3 increases in breathing frequency and 5 changes in tidal volume). With regard to the 5 changes in tidal volume, it was increased from $500 \mathrm{~mL}$ to $600 \mathrm{~mL}$ for 2 subjects, while tidal volume was decreased for 3 subjects. Of the 3 
subjects for whom tidal volume was decreased, there were no concomitant changes in respiratory frequency. Acidemia was present on the initial blood gas for 70 subjects $(24.0 \%)$, of whom only 12 had any ventilator changes made (Table 3).

For subjects who received lung-protective ventilation versus non-lung-protective ventilation, there was no difference in the median values of $\mathrm{pH}$ (7.31 vs 7.30, $P=.82)$ or $\mathrm{P}_{\mathrm{CO}_{2}}$ (44 vs $42, P=.26$ ).

\section{Discussion}

Although blood gases should not be checked routinely for all critically ill patients, ${ }^{17,18}$ blood gas analysis is indicated with changes in respiratory status and initiation of mechanical ventilation. ${ }^{14,17}$ In-patient blood gases frequently result in changes in clinical management, ${ }^{19}$ but the use of blood gases in mechanically ventilated emergency department patients has not been previously described. While a majority of ventilated subjects received some blood gas analysis in this cohort, blood gases were not universal, and the blood gas data do not correlate with ventilator management in the emergency department.

While VBGs are valuable in the emergency department to determine acid/base status for many indications, ${ }^{12,13,20}$ at least one $\mathrm{ABG}$ is indicated in mechanically ventilated patients. ${ }^{14}$ Although $\mathrm{S}_{\mathrm{pO}_{2}}$ is an accurate reflection of $\mathrm{P}_{\mathrm{aO}}$ at physiologic levels, $\mathrm{S}_{\mathrm{pO}_{2}}$ is less reliable in cases of shock ${ }^{21-23}$ or hypoxemia with a $\mathrm{P}_{\mathrm{aO}_{2}}$ of $\leq 60,{ }^{24}$ and $\mathrm{S}_{\mathrm{pO}_{2}}$ cannot provide information about hyperoxia. In this study, the use of ABGs correlated with changes in ventilator settings, whereas use of VBGs or no gas at all did not. While this association may reflect understanding of the need for monitoring ventilated patients by some clinicians, the specific $A B G$ results did not translate to anticipated clinical responses in adjusting ventilator settings.

In this cohort, subjects intubated for respiratory failure did not have ABGs checked more frequently than subjects intubated for other indications. Conversely, those intubated for trauma were more likely to have ABGs than those intubated for other indications. Studies of trauma subjects have found the base deficit from ABGs correlates with outcomes ${ }^{25,26}$ and is useful to guide resuscitation..$^{27,28}$ Moreover, hemoglobin levels, an important clinical data point in the trauma population, can be evaluated quickly at bedside using ABG analyzers. Therefore, the differential use of ABGs in this subgroup may have been influenced by the trauma teams' involvement. In addition, subjects intubated for trauma were significantly younger than subjects intubated for other indications, and after including age in regression model, the observation of increased frequency of ABGs being obtained in trauma subjects lost significance. These findings indicate the need for further studies to determine whether patient age or designation as a trauma victim is a significant predictor of obtaining ABGs in the emergency department.

With increasing emergency department boarding times, ${ }^{29}$ emergency department physicians must manage mechanically ventilated patients for increasingly longer periods. ${ }^{30}$ Despite $>4 \mathrm{~h}$ of ventilation in the emergency department, only $20 \%$ of subjects in this cohort had a change in ventilator settings. Interestingly, subjects without any changes had a longer emergency department LOS than those with changes, and subjects without any blood gases also had longer emergency department LOS than those with blood gases. These findings indicate that lack of time was not the cause for the lack of monitoring or ventilator changes.

The values selected for hypoxemia, ${ }^{24}$ hyperoxia, ${ }^{31-35}$ acidemia, and hypercapnia were chosen at extreme ranges used in the literature to ensure that the values were unambiguous or not subject to clinical context. Additionally, as the cut-offs for acidemia and hypercapnia were the same for arterial and venous gases, we selected clinically relevant values that would indicate the need for action regardless of arterial or venous origin.

Hypoxemia was not associated with ventilator changes. All subjects who were hypoxemic were ventilated with a PEEP of $5 \mathrm{~cm} \mathrm{H}_{2} \mathrm{O}$ and $\mathrm{F}_{\mathrm{IO}_{2}}$ of 1.0 without any titration of ventilator settings for the duration of the emergency department stay. Additionally, the majority of subjects with hyperoxia and $\mathrm{F}_{\mathrm{IO}_{2}}$ of 1.0 had no decrease in the $\mathrm{F}_{\mathrm{IO}_{2}}$ while in the emergency department. Although limited by the heterogeneity of existing studies, hyperoxia has been associated with increased mortality, ${ }^{10}$ duration of mechanical ventilation, length of hospital stay, and subsequent deterioration of oxygenation. ${ }^{8-10}$ Although the data are mixed, ${ }^{31,33,35,36}$ no study has shown improved outcomes with hyperoxia.

This cohort also demonstrated no correlation between acidemia or hypercarbia and changes in tidal volume or breathing frequency. In some instances, the tidal volume was decreased without a concomitant increase in breathing frequency. These findings indicate that overall, while many blood gases were checked, the results were not used to modify oxygenation or ventilation in the emergency department.

Previous surveys of ICU clinicians have found that concerns regarding hypercapnia and acidosis have been barriers to initiating lung-protective ventilation. ${ }^{11}$ In this study, acidemia and hypercarbia did not affect the use of lungprotective ventilation versus non-lung-protective ventilation, further supporting the conclusion that ABG values were not used to guide mechanical ventilation management.

There are limitations to our study, many of which are inherent to its observational nature, as only correlations can be identified without determination of causation. Al- 
though we describe the pattern of blood gas utilization and of changes in mechanical ventilation, we are unable to determine what factors may have influenced clinicians' management with regard to ordering blood gases or making ventilator changes in response to blood gas results. For subjects transferred from another hospital while intubated, a blood gas may have been performed prior to arrival at the tertiary care emergency department. If a blood gas were performed, it is possible that the emergency physician may have not requested another blood gas if there were no change in clinical condition. Despite being a prospective study, not all subjects had times of intubation recorded. In addition, we describe clinical management and subjects' outcomes exclusively from the subjects' emergency department course, and not more longitudinal outcomes. The low frequency of ventilator changes in ventilated subjects in the emergency department may have influenced correlative associations as well, although the rarity of ventilator changes may independently highlight the need for increased attention to ventilated patients in the emergency department. Furthermore, limited data on subjects' comorbidities precluded a more comprehensive adjustment for subject-level parameters that may have affected the frequency of blood gas monitoring or secondary outcomes. Finally, situational parameters such as emergency department census, emergency department capacity, emergency department acuity, subject-to-provider ratios, and hospital census were not assessed and were not included in this study.

Despite these limitations, this study demonstrates the opportunity for future interventions for quality improvement in mechanically ventilated patients in the emergency department. Mechanical ventilation protocols focused on the emergency department, including monitoring of ABGs and recommending subsequent ventilator changes, may be helpful in optimizing PEEP, minimizing exposure to hyperoxia, managing respiratory acidosis, and increasing the adherence to lung-protective ventilation when possible.

\section{Conclusion}

In this cohort of mechanically ventilated subjects, the majority had a blood gas checked while in the emergency department. While ABGs were associated with having changes made to ventilator settings in the emergency department, clinical findings of hypoxemia, hyperoxia, hypercapnia, and acidemia were not. Inattention to blood gas results may lead to missed opportunities in guiding ventilator changes in the emergency department.

\section{REFERENCES}

1. Wunsch H, Linde-Zwirble WT, Angus DC, Hartman ME, Milbrandt EB, Kahn JM. The epidemiology of mechanical ventilation use in the United States. Crit Care Med 2010;38(10):1947-53.

2. Modrykamien AM, Killian L, Walters RW. Liberal manipulation of ventilator settings and its impact on tracheostomy rate and ventilatorfree days. Respir Care 2016;61(1):30-35.

3. Rose L, Scales DC, Atzema C, Burns KE, Gray S, Doing C, et al. Emergency department length of stay for critical care admissions: a population-based study. Ann Am Thorac Soc 2016;13(8):1324-32.

4. Fuller BM, Mohr NM, Miller CN, Deitchman AR, Levine BJ, Castagno N, et al. Mechanical ventilation and ARDS in the emergency department: a multicenter, observational, prospective, cross-sectional study. Chest 2015;148(2):365-74.

5. Wilcox SR, Richards JB, Fisher DF, Sankoff J, Seigel TA. Initial mechanical ventilator settings and lung protective ventilation in the emergency department. Am J Emerg Med 2016;34(8):1446-51.

6. Bhat R, Goyal M, Graf S, Bhooshan A, Teferra E, Dubin J, Frohna B. Impact of post-intubation interventions on mortality in patients boarding in the emergency department. West J Emerg Med 2014; 15(6):708-11.

7. Wood S, Winters ME. Care of the intubated emergency department patient. J Emerg Med 2011;40(4):419-27.

8. Rachmale S, Li G, Wilson G, Malinchoc M, Gajic O. Practice of excessive $\mathrm{F}(\mathrm{IO}(2))$ and effect on pulmonary outcomes in mechanically ventilated patients with acute lung injury. Respir Care 2012; 57(11):1887-93.

9. Helmerhorst HJ, Roos-Blom MJ, van Westerloo DJ, de Jonge E. Association between arterial hyperoxia and outcome in subsets of critical illness: a systematic review, meta-analysis, and meta-regression of cohort studies. Crit Care Med 2015;43(7):1508-19.

10. Damiani E, Adrario E, Girardis M, Romano R, Pelaia P, Singer M, Donati A. Arterial hyperoxia and mortality in critically ill patients: a systematic review and meta-analysis. Crit Care 2014;18(6):711.

11. Rubenfeld GD, Cooper C, Carter G, Thompson BT, Hudson LD. Barriers to providing lung-protective ventilation to patients with acute lung injury. Crit Care Med 2004;32(6):1289-93.

12. Kelly AM. Can VBG analysis replace ABG analysis in emergency care? Emerg Med J 2016;33(2):152-54.

13. Bloom BM, Grundlingh J, Bestwick JP, Harris T. The role of venous blood gas in the emergency department: a systematic review and meta-analysis. Eur J Emerg Med 2014;21(2):81-88.

14. Davis MD, Walsh BK, Sittig SE, Restrepo RD. AARC clinical practice guideline: blood gas analysis and hemoximetry: 2013. Respir Care 2013;58(10):1694-703.

15. ARDS Definition Task Force, Ranieri VM, Rubenfeld GD, Thompson BT, Ferguson ND, Caldwell E, et al. Acute respiratory distress syndrome: the Berlin definition. JAMA 2012;307(23):2526-33.

16. Villar J, Pérez-Méndez L, Blanco J, Añón JM, Blanch L, Belda J, et al. A universal definition of ARDS: the $\mathrm{PaO} / \mathrm{FiO} 2$ ratio under a standard ventilatory setting: a prospective, multicenter validation study. Intensive Care Med 2013;39(4):583-92.

17. Martinez-Balzano CD, Oliveira P, O'Rourke M, Hills L, Sosa AF, Critical Care Operations Committee of the UMass Memorial Healthcare Center. An educational intervention optimizes the utilization of arterial blood gases across intensive care units from different specialties: a quality improvement study. Chest 2017;151(3):579-85.

18. Blum FE, Lund ET, Hall HA, Tachauer AD, Chedrawy EG, Zilberstein J. Reevaluation of the utilization of arterial blood gas analysis in the intensive care unit: effects on patient safety and patient outcome. J Crit Care 2015;30(2):438.e1-438.e5.

19. Melanson SE, Szymanski T, Rogers SO, Jarolim P, Frendl G, Rawn JD, et al. Utilization of arterial blood gas measurements in a large tertiary care hospital. Am J Clin Pathol 2007;127(4):604-09. 


\section{Ventilator Changes in the Emergency Department}

20. Byrne AL, Bennett M, Chatterji R, Symons R, Pace NL, Thomas PS. Peripheral venous and arterial blood gas analysis in adults: are they comparable? A systematic review and meta-analysis. Respirology 2014;19(2):168-75.

21. Van de Louw A, Cracco C, Cerf C, Harf A, Duvaldestin P, Lemaire $\mathrm{F}$, Brochard L. Accuracy of pulse oximetry in the intensive care unit. Intensive Care Med 2001;27(10):1606-1613.

22. Perkins GD, McAuley DF, Giles S, Routledge H, Gao F. Do changes in pulse oximeter oxygen saturation predict equivalent changes in arterial oxygen saturation? Crit Care 2003;7(4):R67.

23. O'Driscoll BR, Howard LS, Davison AG, British Thoracic Society. Emergency oxygen use in adult patients: concise guidance. Clin Med (Lond) 2011;11(4):372-75.

24. Zeserson E, Goodgame B, Hess JD, Schultz K, Hoon C, Lamb K, et al. Correlation of venous blood gas and pulse oximetry with arterial blood gas in the undifferentiated critically ill patient. J Intensive Care Med 2016 [Epub ahead of print] doi: 10.1177/0885066616652597.

25. Davis JW, Kaups KL. Base deficit in the elderly: a marker of severe injury and death. J Trauma 1998;45(5):873-77.

26. Rutherford EJ, Morris JA Jr., Reed GW, Hall KS. Base deficit stratifies mortality and determines therapy. J Trauma 1992;33(3):417-23.

27. Davis JW, Shackford SR, Mackersie RC, Hoyt DB. Base deficit as a guide to volume resuscitation. J Trauma 1988;28(10):1464-67.

28. Davis JW, Kaups KL, Parks SN. Base deficit is superior to $\mathrm{pH}$ in evaluating clearance of acidosis after traumatic shock. J Trauma 1998;44(1):114-18.

29. Institute of Medicine. IOM report: the future of emergency care in the United States health system. Acad Emerg Med 2006;13(10): 1081-85.
30. Mudumbai SC, Barr J, Scott J, Mariano ER, Bertaccini E, Nguyen H, et al. Invasive mechanical ventilation in California over 2000-2009: implications for emergency medicine. West J Emerg Med 2015; 16(5):696-706

31. Kilgannon JH, Jones AE, Shapiro NI, Angelos MG, Milcarek B, Hunter K, et al.; on behalf of the Emergency Medicine Shock Research Network (EMShockNet) Investigators. Association between arterial hyperoxia following resuscitation from cardiac arrest and in-hospital mortality. JAMA 2010;303(21):2165-71.

32. Bellomo R, Bailey M, Eastwood GM, Nichol A, Pilcher D, Hart GK, et al.; on behalf of the Study of Oxygen in Critical Care (SOCC) Group. Arterial hyperoxia and in-hospital mortality after resuscitation from cardiac arrest. Crit Care 2011;15(2):R90.

33. Ihle JF, Bernard S, Bailey MJ, Pilcher DV, Smith K, Scheinkestel $\mathrm{CD}$. Hyperoxia in the intensive care unit and outcome after out-ofhospital ventricular fibrillation cardiac arrest. Crit Care Resusc 2013; 15(3):186-90.

34. Nelskyla A, Parr MJ, Skrifvars MB. Prevalence and factors correlating with hyperoxia exposure following cardiac arrest: an observational single centre study. Scand J Trauma Resusc Emerg Med 2013;21:35.

35. Rincon F, Kang J, Maltenfort M, Vibbert M, Urtecho J, Athar MK, et al. Association between hyperoxia and mortality after stroke: a multicenter cohort study. Crit Care Med 2014;42(2):387-96.

36. Panwar R, Hardie M, Bellomo R, Barrot L, Eastwood GM, Young PJ, et al.; on behalf of the CLOSE Study Investigators and ANZICS Clinical Trials Group. Conservative versus liberal oxygenation targets for mechanically ventilated patients. a pilot multicenter randomized controlled trial. Am J Respir Crit Care Med 2016;193(1):43-51.

This article is approved for Continuing Respiratory Care Education credit. For information and to obtain your CRCE

(free to AARC members) visit

www.rcjournal.com

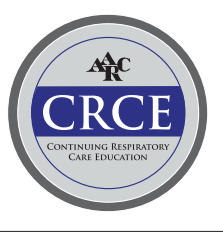

